\title{
A fully dispersive fifth order nonlinear wave model. I: Theoretical part
}

\author{
${ }^{a}$ Y. ZHANG \& W.B. FENG, ${ }^{b}$ X.Q. JI, ${ }^{c}$ M.M. WANG \\ ${ }^{a}$ College of Harbour, Coastal and Offshore Engineering, Hohai University, Nanjing, \\ Jiangsu,P.R.C \\ ${ }^{b}$ Jiangsu Provincial Water Transport Engineering Technique Research Center, Nanjing, \\ Jiangsu,P.R.C \\ 'Jiangsu Province Engineering Consulting Center, Nanjing, Jiangsu,P.R.C
}

KEYWORD: fully dispersive; nonlinear; wave propagation model; mild slope equations; Boussinesqtype equations

ABSTRACT: A fully dispersive fifth order nonlinear wave propagation model for mild current, water level and depth was established theoretically considering energy and topography factors. It satisfied both the frequency dispersion and nonlinearity. By omitting the $5^{\text {th }}$ order terms, the lower order model was in consistency with the former 3rd order model. This model could be simplified to mildslope type equations for deep water, to Boussinesq type equations for shallow water and to Airy wave for very shallow water.

\section{INTRODUCTION}

Wave is an important ocean dynamic factor. It has great influence on maritime structures safety, harbor ship berthing, sediment transport and pollutant dispersion. In the last two decades, wave propagation model developed in two main direction: 1) fully dispersive mild slope equation and its extension 2) weakly dispersive nonlinear Boussinesq-type equation and its extension. The Boussinesq-type equations are weakly dispersive nonlinear equations, suitable for shallow water and has limitations in calculating wave propagation in deep water. Though some extensions of B-Type equations improved the limitation to water depth, the situations for water depth equivalent to or larger than wave length, the results of extension models were still not satisfied. The mild slope equation is suitable for modeling linear single frequency and has limitations in shallow water wave propagation in near shore areas. Though the time-related mild-slope equation can solved the frequency problem, the band-width of spectrum was still limited. To breakthrough this limitations, the researchers proposed the mathematical models which satisfied fully dispersion and nonlinearity simultaneously. The calculation model and the applicability are yet to be discussed. Nadaoka (1994) et al adopted vertical dependent function to express wave velocity, and by applying Galerkin method, these vertical dependent functions were idealized combined. Those functions could be used to express any wave velocity field and finally a called fully dispersive nonlinear mathematical model was achieved. Isobe (1994) approximated the frequency related coefficient as rational function, and extend the original mild slope equations into a "time-related mild slope equation" for random waves. It can depict time evolution process of near shore short waves; Li B. (2008) adopted vertical characteristic horizontal velocity to deduce control functions. The most improvement of the new control function was that it could satisfy the dispersion relation in the range of full water, and the linear shallow gradient factor is in good agreement with Stokes theoretical solution. The authors concluded that this model can simulate wave propagating from shallow water to deep water $(k h=0 \sim 4)$, and can be simplified to Nwogu equations by ignoring the high order terms (higher than $\left.(k h)^{4}\right)$. Hong et al (2009) deduced a nonlinear mathematic model based on gravity surface wave and long wave nonlinear interaction. This model is based on non-viscosity, non-rotational fluid dynamic equations. It is a new fully dispersion and nonlinear full water equation for mild slope, water elevation and current. These governing equations contain energy coefficient reflecting energy input, friction and wave breaking loss and topography coefficient reflecting the local depth variation. This model suits wave propagating from deep water to very shallow water, with long wave field and water depth varied. Zhang et al (2010a) extended it into third order and third order modified model (2010b). This paper aims to deduce a fifth order theoretical model. 


\section{THEORETICAL MODEL DERIVATION}

Assuming the flow is irrotational, the continuity equation can be expressed in linear Laplace equation:

$\nabla^{2} \phi_{T}+\frac{\partial^{2} \phi_{T}}{\partial z^{2}}=0,-h<\eta<\eta_{T}$

where $\nabla \equiv(\partial / \partial x, \partial / \partial y)$ is horizontal gradient operator; $x, y$ are horizontal directions; $z$ directs vertically; $\eta_{T}(x, y, t)$ is free surface elevation; $z=0$ is still water level; $z=-h(x, y)$ is bottom boundary.

On free surface elevation $z=\eta_{T}$ it satisfy kinematic equation, and can be expressed as:

$$
\frac{\partial \eta_{T}}{\partial \mathrm{t}}+\nabla \varphi_{T} \cdot \nabla \eta_{T}=\frac{\partial \varphi_{T}}{\partial z}, z=\eta_{T}
$$
face.

Unsteady Bernoulli equations can be used to depict the dynamic boundary conditions on free sur-

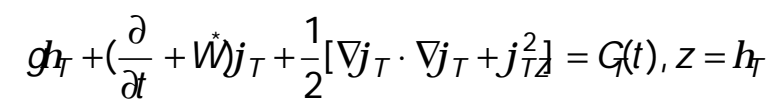

where $g$ is gravitational acceleration; $W^{*}$ is composed energy factor, representing energy dissipation coefficient.

Substituting Eq.(3) into (2), a new kinematic-dynamic boundary condition can be deduced:

$g \frac{\partial \varphi_{T}}{\partial z}+\left(\frac{\partial}{\partial t}+W^{*}\right)\left[\frac{\partial \varphi_{T}}{\partial t}+\left(\nabla \varphi_{T} \cdot \nabla \varphi_{T}+\varphi_{T Z}^{2}\right)\right]$

$+\frac{1}{2} \nabla \varphi_{T} \cdot \nabla\left(\nabla \varphi_{T} \cdot \nabla \varphi_{T}+\varphi_{T Z}^{2}\right)$

$+\frac{1}{2} \varphi_{T Z} \frac{\partial}{\partial z}\left(\nabla \varphi_{T} \cdot \nabla \varphi+\varphi_{T Z}^{2}\right)=0, z=\eta_{T}$

Since Eq.(1) is linear, $\varphi_{T}$ can be divided into wave velocity potential $\varphi$ and long wave velocity potential $\varphi_{\mathrm{c}}$ :

$$
\varphi_{T}=\varphi(x, y, z, t)+\varphi_{c}(x, y, z, t)
$$

Here are two assumptions: 1) the vertical acceleration was omitted, i.e. $\left.\partial^{2} \varphi c / \partial z^{2}=0 ; 2\right)$ the vertical wave velocity was also omitted, i.e. $\partial \varphi_{c} \partial z=0$. Adopting the above two assumptions into the equations, only the horizontal velocity $U c=\nabla \varphi_{c}$ exist.

The wave elevation $\eta_{T}$ can be divided into the following form:

$$
\eta_{\mathrm{T}}=\eta+\eta_{\mathrm{c}}
$$

To convert the 3 dimensional control Eq.s(1)-(5) into horizontal 2 dimensions, the $3 \mathrm{~d}$ velocity potential function $\varphi(x, y, z, t)$ can be divided into local part $F(x, y, z, t)$ and removal part $\Phi(x, y, t)$. The local part depicts the water local change and the removal part depicts the overall change of horizontal water area. Assuming the wave velocity potential as:

$\varphi(x, y, z, t)=F(x, y, z, t) \Phi(x, y, t)$

Using the second order Green Function:

$$
\int_{-h}^{\eta_{T}}\left(F \varphi_{z z}-\varphi F_{z z}\right) d z=\left.\left(F \varphi_{z}-\varphi F_{z}\right)\right|_{-h} ^{\eta_{T}}=0
$$

Wave can be divided from current in Eq.s (1)

$$
\varphi_{\mathrm{zz}}=-\nabla^{2} \varphi-\nabla^{2} \varphi_{\mathrm{c}}=-\nabla \cdot \nabla \varphi-\nabla \cdot \mathrm{U}_{\mathrm{c}}
$$

Substituting Eq. (9) into Eq. (8), we can get 
$\int_{-h}^{\eta_{T}}\left(F\left(\nabla \cdot \nabla \varphi+\nabla \cdot U_{c}\right)+\varphi F_{z z}\right) d z=\left.\left(\varphi F_{z}-F \varphi_{z}\right)\right|_{-h} ^{n_{T}}=0$

Substituting $\varphi F_{z z}=F \varphi_{z z}$ into Eq. (10), we can get

$\int_{-h}^{\eta_{T}} F\left(\nabla \cdot \nabla \phi+\nabla \cdot U_{c}+\phi_{z z}\right) d z=0$

The Eq. (11) is an integration of 3 dimension continuity equation from bottom to free surface. $F$ is weight factor.

Adopting the following form

$\mathrm{F} \nabla \cdot \nabla \varphi=\mathrm{F} \nabla \cdot(\mathrm{F} \nabla \Phi+\Phi \nabla \mathrm{F})=\nabla \cdot\left(\mathrm{F}^{2} \nabla \Phi\right)+\Phi \mathrm{F} \nabla \cdot \overrightarrow{\mathrm{F}}$

and

$\int_{-h}^{\eta_{T}} F \nabla \cdot \nabla \varphi d z=\left.\left[\nabla \cdot \int_{-h}^{\eta_{T}} F^{2} \nabla \Phi d z-F^{2} \nabla \Phi \cdot \nabla Z\right]\right|_{-h} ^{\eta_{T}}$

$+\Phi \int_{-h}^{\eta_{T}} \mathrm{~F} \nabla \cdot \nabla \mathrm{Fdz}$

Eq. (10) can be expressed as:

$\nabla \cdot \int_{-h}^{\eta_{T}} F^{2} \nabla \Phi d z+\int_{-h}^{n_{T} T}\left[F \nabla \cdot U_{c}+\Phi F_{z z}\right] d z$

$+\Phi \int_{-h}^{n_{T}} F \nabla \cdot \nabla F d z-\left.F^{2} \nabla \Phi \cdot \nabla Z\right|_{-h} ^{n_{T}}=\left.\left(\Phi F_{z}-F_{z}\right)\right|_{-h} ^{n_{T}}=0$

Adopting the kinematic boundary condition at $z=\eta_{T}$ and $z=-h$, we can get :

$-\left.\left\{\mathrm{F}^{2} \nabla \Phi \cdot \nabla Z+\left(\Phi \mathrm{F} \mathrm{F}_{\mathrm{Z}}-\mathrm{F} \varphi_{\mathrm{z}}\right)\right\}\right|_{-h} ^{\eta_{\mathrm{T}}}$

$=\left.\left\{\left.\mathrm{F}\right|^{\eta_{T}} \frac{\mathrm{D} \eta}{\mathrm{Dt}}-\Phi \mathrm{F} \mathrm{F}_{\mathrm{Z}}+\Phi \mathrm{F} \nabla \mathrm{F} \cdot \nabla \mathrm{ZZ}\right\}\right|_{-\mathrm{h}} ^{\eta_{\mathrm{T}}}$

$+\left[\left.F\right|^{\eta_{T}} \frac{D \eta_{c}}{D t}+\left.F\right|_{-h}\left(\overrightarrow{U_{c}} \cdot \nabla h\right)\right]$

Substituting into (10), the following form can be obtained:

$\left.F\right|^{\eta_{T}} \frac{D \eta}{D t}+\nabla \cdot\left[\int_{-h}^{\eta_{T}} F^{2} d z\right] \nabla \Phi+\left(\nabla \cdot U_{c}\right)\left[\int_{-h}^{\eta_{T}} F d z-\beta\right]$

$+\left[\int_{-h}^{\eta_{T}} F F_{z z} d z-\left.F F_{z}\right|^{\eta_{T}}+J / g\right] \Phi$

$=-\left[\left.F\right|^{\eta_{T}} \frac{D \eta_{c}}{D t}+\left.F\right|_{-h}\left(\vec{U}_{c} \cdot \nabla h\right)+\beta \nabla \cdot U_{c}\right]=0$

where

$J / g=\int_{-h}^{\eta_{T}} F \nabla \cdot \nabla F d z+\left.F \nabla F \cdot \nabla Z\right|_{-h} ^{\eta_{T}}+\left.F F_{z}\right|_{-h}$

By adopting the free surface dynamic boundary condition $z=\eta_{T}$ and substituting the Eq. (7) into (3), the free surface boundary condition can be changed to:

$g \eta+\left(\frac{D}{D t}+W^{*}\right) \varphi+\frac{1}{2}\left[\nabla \varphi \cdot \nabla \varphi+\varphi_{z}^{2}\right]=C(t)$

By adopting $\varphi=F \Phi$, another 2D control Equation can be achieved:

$g \eta+[F]\left(\frac{D}{D t}+W^{*}\right) \Phi+\left[\frac{D F}{D t}\right] \Phi+\frac{1}{2}\left[F^{2}(\nabla \Phi \cdot \nabla \Phi)\right.$

$\left.+\left(\mathrm{F}_{\mathrm{z}}{ }^{2}+\nabla \mathrm{F} \cdot \nabla \mathrm{F}\right) \Phi^{2}+\Phi \nabla \Phi \cdot \nabla \mathrm{F}^{2}\right]=\mathrm{C}(\mathrm{t})$

or can be expressed as 
$g \eta+[F] \frac{D \Phi}{D t}+\left(\frac{D F}{D t}+W^{*} F\right) \Phi+\frac{1}{2}\left[F^{2}(\nabla \Phi \cdot \nabla \Phi)\right.$

$\left.+\left(\mathrm{F}_{\mathrm{z}}{ }^{2}+\nabla \mathrm{F} \cdot \nabla \mathrm{F}\right) \Phi^{2}+\left(\nabla \mathrm{F}^{2} \cdot \nabla \Phi\right) \Phi\right]=\mathrm{C}(\mathrm{t}), \mathrm{z}=\eta_{\mathrm{T}}$

Eq.s(15),(19) are governing equation of shallow water wave propagation model on long waves. $\Phi, \eta$ are nonlinear wave velocity potential and wave surface; $\Phi c, \eta c$ and $U$ are given wave velocity potential, wave surface and horizontal velocity component; $h$ is depth under still water level, $g$ is gravitational acceleration. To get explicit expression, the following expression was adopted

$F(x, y, z, t)=\cosh k(h+z) / \cosh k\left(h+\eta_{c}\right)$,

$-h \leq z \leq \eta_{T}=\eta+\eta_{c}$

Dispersion coefficient $k$ can be determined by Eq. (21)

$\mathrm{gk} \gamma=\mathrm{gk} \tanh \mathrm{kd}=\sigma^{2}=\sigma^{2}\left[1+\left(\frac{\mathrm{W}^{*}}{2 \sigma}\right)^{2}\right] \approx \sigma^{2}$

where $\sigma$ stands for natural frequency

$$
\begin{aligned}
& \sigma=\left(\omega-\stackrel{r}{K} \cdot U_{c}\right)=-\frac{D}{D t} \Psi(x, y, t) \\
& \omega=-\frac{\partial}{\partial t} \Psi=\sigma+\stackrel{r}{K} \cdot \stackrel{r}{U}_{c}^{r}=-\left(\Phi_{1} \frac{\partial \Phi_{2}}{\partial t}-\Phi_{2} \frac{\partial \Phi_{1}}{\partial t}\right) /|\Phi|^{2} \\
& \stackrel{K}{\prime}=\nabla \Psi=\left(\Phi_{1} \nabla \Phi_{2}-\Phi_{2} \nabla \Phi_{1}\right) /|\Phi|^{2} \\
& \Phi=R e \Phi+\operatorname{Im} \Phi=\Phi_{1}+i \Phi_{2}=\Phi \mid e^{i \Psi} \\
& |\Phi|^{2}=\Phi_{1}^{2}+\Phi_{2}^{2}, \Psi=\operatorname{arctg}\left(\Phi_{2} / \Phi_{1}\right)
\end{aligned}
$$

Adopting (20), Eq.s (15), (19) can be expressed as follows:

$A_{1} \frac{D \eta}{D t}+\nabla \cdot\left(A_{3} \nabla \Phi\right)+A_{2} \nabla \dot{U}_{c}^{r}+A_{4} \Phi=0, Z=\eta_{T}$

$g \eta+B_{1} \frac{D \Phi}{D t}+\left[\left(\frac{D}{D t}+W^{*}\right) B_{1}+B_{4} \cdot \nabla \Phi\right] \Phi$

$+\frac{1}{2}\left[B_{2}(\nabla \Phi \cdot \nabla \Phi)+B_{3} \Phi^{2}\right]=C(t), z=\eta_{T}$

where the coefficients are:

$$
\begin{aligned}
& A=\left.F\right|^{\eta_{T}}=B=\cosh k(d+\eta) / \cosh k d \\
& \cong 1+\gamma(k \eta)+\frac{1}{2}(k \eta)^{2}+\frac{1}{6} \gamma(k \eta)^{3}+\frac{1}{24}(k \eta)^{4}+\frac{1}{120} \gamma(k \eta)^{5} \\
& =1+\frac{8 \%}{g} \eta+\frac{1}{2}(k \eta)^{2}+\frac{1}{6} \frac{8 \%}{g} k^{2} \eta^{3}+\frac{1}{24}(k \eta)^{4}+\frac{1}{120} \frac{8 \%}{g} k^{4} \eta^{5} \\
& =B
\end{aligned}
$$




$$
\begin{aligned}
& A_{2}=\int_{-h}^{\eta T} F d z-\beta=\frac{\sinh k(d+\eta)}{k \cosh k d}-\beta, \beta=\gamma / k \\
& \cong \eta+\frac{1}{2} \gamma k \eta^{2}+\frac{1}{6} k^{2} \eta^{3}+\frac{1}{24} \gamma^{3} \eta^{4}+\frac{1}{120} k^{4} \eta^{5} \\
& =\eta+\frac{1}{2} \frac{\sigma^{2}}{g} \eta^{2}+\frac{1}{6} k^{2} \eta^{3}+\frac{1}{24} \frac{\sigma^{2}}{g} k^{2} \eta^{4}+\frac{1}{120} k^{4} \eta^{5} \\
& \left.A_{3}=\int_{-h}^{\pi} F^{2} d z=[2 k(d+\eta)+\sinh 2 d+\eta)\right] /\left(4 k \cosh h^{2} k d\right) \\
& \cong \frac{\epsilon \epsilon_{g}}{g}+\eta+\gamma k \eta^{2}+\frac{1}{3}\left(1+\gamma^{2}\right) k^{2} \eta^{3}+\frac{1}{3} \gamma k^{3} \eta^{4}+\frac{1}{15}\left(1+\gamma^{2}\right) k^{4} \eta^{5} \\
& =\frac{G \epsilon_{g}}{g}+\eta+\frac{\sigma^{2}}{g} \eta^{2}+\frac{1}{3}\left[k^{2}+\left(\frac{\sigma^{2}}{g}\right)^{2} \eta^{3}\right. \\
& +\frac{1}{3} \frac{\sigma^{2}}{g} k^{2} \eta^{4}+\frac{1}{15}\left(k^{2}+\left(\frac{\sigma^{2}}{g}\right)^{2}\right) k^{2} \eta^{5}
\end{aligned}
$$

$$
A_{4}=A_{41}+A_{42}+J / g
$$

$$
A_{41}=\int_{-h}^{\eta_{T}} F F_{z z} d z=k^{2} A_{3}
$$

$$
A_{42}=-\left.F F_{z}\right|^{\eta_{T}}=-k \sinh 2 k(d+\eta) /\left(2 \cosh ^{2} k d\right)
$$$$
\cong-\left\{k \gamma+\left(1+\gamma^{2}\right) k^{2} \eta+2 \gamma k^{3} \eta^{2}+\frac{2}{3}\left(1+\gamma^{2}\right) k^{4} \eta^{3}\right.
$$$$
\left.+\frac{2}{3} k^{5} \eta^{4}+\frac{2}{15}\left(1+\gamma^{2}\right) k^{6} \eta^{5}\right\}
$$

$$
=-\left\{\frac{\sigma^{2}}{g}+\left[k^{2}+\left(\frac{\sigma^{2}}{g}\right)^{2}\right] \eta+2 \frac{\sigma^{2}}{g}(k \eta)^{2}+\frac{2}{3}\left[k^{2}\right.\right.
$$$$
\left.\left.+\left(\frac{\sigma^{2}}{g}\right)^{2}\right] k^{2} \eta^{3}+\frac{2}{3} \frac{\sigma^{2}}{g}(k \eta)^{4}+\frac{2}{15}\left[k^{2}+\left(\frac{\sigma^{2}}{g}\right)^{2}\right] k^{4} \eta^{5}\right\}
$$

$J / g=\int_{-h}^{\eta_{T}} F \nabla \cdot \nabla F d z+\left.F \nabla F \cdot \nabla Z\right|_{-h} ^{\eta_{T}}+\left.F F_{z}\right|_{-h}$

$B_{2}=\left.F^{2}\right|^{\eta_{T}}=[1+\cosh 2 k(d+\eta)] / 2 \cos ^{2} k d$

$$
\cong 1+2 \gamma k \eta+\left(1+\gamma^{2}\right)(k \eta)^{2}+\frac{4}{3} \gamma(k \eta)^{3}
$$

$$
+\frac{1}{3}\left(1+\gamma^{2}\right)(k \eta)^{4}+\frac{4}{15} \gamma(k \eta)^{5}
$$

$$
\begin{aligned}
= & 1+2 \frac{\sigma^{2}}{g} \eta+\left[k^{2}+\left(\frac{\sigma^{2}}{g}\right)^{2}\right] \eta^{2}+\frac{4}{3} \frac{\sigma^{2}}{g} k^{2} \eta^{3} \\
& +\frac{1}{3}\left[k^{2}+\left(\frac{\sigma^{2}}{g}\right)^{2}\right] k^{2} \eta^{4}+\frac{2}{15} \frac{\sigma^{2}}{g} k^{4} \eta^{5}
\end{aligned}
$$

$B_{3}=B_{31}+B_{32}$ 


$$
\begin{aligned}
& B_{31}=\left.F_{z}^{2}\right|^{\eta_{T}}=k^{2}[\cosh 2 k(d+\eta)-1] / 2 \cos ^{2} k d \\
& \cong(k \gamma)^{2}+2 k^{3} \eta+\left(1+\gamma^{2}\right) k^{4} \eta^{2}+\frac{4}{3} k^{5} \eta^{3}+\frac{1}{3}\left(1+\gamma^{2}\right) k^{6} \eta^{4}+\frac{4}{15} k^{7} \eta^{5} \\
& =\left(\frac{\sigma^{2}}{g}\right)^{2}+2 \frac{\sigma^{2}}{g} k^{2} \eta+\left[k^{2}+\left(\frac{\sigma^{2}}{g}\right)^{2}\right](k \eta)^{2}+\frac{4}{3} \frac{\sigma^{2}}{g} k^{4} \eta^{3} \\
& +\frac{1}{3}\left[k^{2}+\left(\frac{\sigma^{2}}{g}\right)^{2}\right](k \eta)+\frac{4}{15} \frac{\sigma^{2}}{g} k^{6} \eta^{5} \\
& \mathrm{~B}_{32}=\left.\sqrt{\mathrm{F}} \cdot \sqrt{\mathrm{F}}\right|^{\eta_{\mathrm{T}}} \\
& =\frac{1}{2 \cosh k d}\{\cosh 2\langle d+\eta)-1][\nabla k(d+\eta) \cdot \nabla k(d+\eta)] \\
& \left.+\gamma^{2}[\cosh 2 k(d+\eta)-1](\nabla k d \cdot \nabla k d)-2 \gamma \sinh 2 k(d+\eta)[\nabla k(d+\eta) \cdot \nabla k d]\right\} \\
& \cong\left\{\gamma^{2}+2 \gamma k \eta+\left(1+\gamma^{2}\right)(k \eta)^{2}+\frac{4}{3} \gamma(k \eta)^{3}+\frac{\left(1+\gamma^{2}\right)}{3}(k \eta)^{4}\right. \\
& \left.\left.+\frac{4}{15} \gamma(k \eta)^{5}\right\}(\nabla k \eta \cdot \nabla k \eta)+21-\gamma^{2}\right) k \eta\left\{\gamma+k \eta+\frac{2}{3} \gamma(k \eta)^{2}+\frac{1}{3}(k \eta)^{3}\right. \\
& \left.+\frac{2}{15} \gamma(k \eta)^{4}\right\}(\nabla k \eta \cdot \nabla k d)+\left(1-\gamma^{2}\right)^{2}(k \eta)^{2}\left\{1+\frac{1}{3}(k \eta)^{2}\right\}(\nabla k d \cdot \nabla k d) \\
& =\left[\left(1-\gamma^{2}\right)^{2}(k \eta)^{2}(\nabla k d \cdot \nabla k d)+2 \gamma\left(1-\gamma^{2}\right) k n(\nabla k d \cdot \nabla k d)+\gamma^{2}(\nabla k \eta \cdot \nabla k \eta)\right] \\
& \left.+\left[\not 1-\gamma^{2}\right)(k \eta)^{2}(\nabla k \eta \cdot \nabla k d)+2 \gamma k m(\nabla k \eta \cdot \nabla k \eta)\right] \\
& +\left[\frac{1}{3}\left(1-\gamma^{2}\right)^{2}(k \eta)^{4}(\nabla k d \cdot \nabla k d)+\frac{4}{3} \gamma\left(1-\gamma^{2}\right)(k \eta)^{3}(\nabla k \eta \cdot \nabla k d)\right. \\
& \left.+\left(1+\gamma^{2}\right)(k \eta)^{2}(\nabla k \eta \cdot \nabla k d)\right]+\left[\frac{2}{3}\left(1-\gamma^{2}\right)(k \eta)^{4}(\nabla k \eta \cdot \nabla k d)\right. \\
& \left.+\frac{4}{3} \gamma(k \eta)^{3}(\nabla k \eta \cdot \nabla k \eta)\right] \\
& \mathrm{B}_{4}=\left.\mathrm{F} \nabla \mathrm{F}\right|^{\eta_{\mathrm{T}}} \\
& =\frac{1}{2 \cosh k d}\{\sinh 2 k(d+\eta) \nabla k(d+\eta)-\gamma(\cosh 2 k(d+\eta)+1] \nabla k d\} \\
& \cong\left\{\gamma+\left(1+\gamma^{2}\right) k \eta+2 \gamma(k \eta)^{2}+\frac{2}{3}\left(1+\gamma^{2}\right)(k \eta)^{3}+\frac{2}{3} \gamma(k \eta)^{4}\right. \\
& \left.+\frac{2}{15}\left(1+\gamma^{2}\right)(k \eta)^{5}\right\} \nabla k \eta+\left(1-\gamma^{2}\right) k \eta\left\{1+\gamma k \eta+\frac{2}{3}(k \eta)^{2}+\frac{\gamma}{3}(k \eta)^{3}\right. \\
& \left.+\frac{2}{15}(\mathrm{k} \eta)^{4}\right\} \nabla k d \\
& =\left[\left(1-\gamma^{2}\right) \mathrm{k} \eta \nabla k d+\gamma \nabla k \eta\right]+k \eta\left[\gamma\left(1-\gamma^{2}\right) k \eta \nabla k d+\left(1+\gamma^{2}\right) \nabla k \eta\right] \\
& +2 k \eta)^{2}\left[\frac{1}{3}\left(1-\gamma^{2}\right) k \eta \nabla k d+\gamma / k \eta\right]+\frac{1}{3}(k \eta)^{3}\left[\gamma\left(1-\gamma^{2}\right) k \eta \nabla k d\right. \\
& \left.\left.+21+\gamma^{2}\right) \nabla k \eta\right]+\frac{2}{3}(k \eta)^{4}\left[\frac{1}{5}\left(1-\gamma^{2}\right) k \eta \nabla k d+\gamma \nabla k \eta\right]
\end{aligned}
$$

The exactness of the mathematical model is determined by the order of the coefficients. Choosing higher order of coefficients yields more accurate model results and it demands high calculation ability. Keep the third order coefficients, we can get former the third order solutions.

\section{SPECIAL CASE ANALYSIS}

This model can be transformed into equivalent nonlinear mild slope equations for slowing varied current, water level and depth and Boussinesq-type shallow water nonlinear wave model for a relative shallow water depth and Airy shallow water Equations.

Nonlinear deep water waves (mild-slope type)

when $k h \gg 1, \sigma^{2} / g=k, c_{g}=c / 2, c c_{g}=g / 2 k, J=0$, the governing equations can be simplified to the mildslope type equations : 
$\left(1+k \eta+\frac{1}{2} k^{2} \eta^{2}\right) \frac{D \eta}{D t}+\left(1+\frac{1}{2} k \eta+\frac{1}{6} k^{2} \eta^{2} \eta \eta \nabla \cdot \vec{U}_{c}\right.$

$+\nabla \cdot\left[\left(\frac{1}{2}+k \eta+k^{2} \eta^{2}\right) \nabla \Phi / k\right]-\left(\frac{1}{2}+k \eta+k^{2} \eta^{2}\right) k \Phi=0$

and

$$
\begin{aligned}
& g \eta+\left(\frac{D}{D t}+W^{*}\right)\left(1+k \eta+\frac{1}{2} k^{2} \eta^{2}\right) \Phi \\
& +\left(\frac{1}{2}+\mathrm{k \eta}\right)\left(\nabla \Phi \cdot \nabla \Phi+\mathrm{k}^{2} \Phi^{2}\right)=\mathrm{C}(\mathrm{t})
\end{aligned}
$$

\section{Shallow water nonlinear long wave equations ( Boussinesq-type )}

When $k h<1, \sigma^{2} / g=k^{2} h\left[1-(k h)^{2} / 3\right], c c_{g}=g h\left[1-2(k d)^{2} / 3\right], \quad k^{2} c c_{g}-\sigma^{2}=-g k^{4} h^{3} / 3, \quad \sigma^{4} / g=g k^{4} h^{2}\left[1-2(k h)^{2} / 3\right]$ on slowly varied current, water level and water depth, approximately take $\nabla \cdot \nabla \Phi=-k^{2} \Phi$, the governing equations could be transformed to Boussinesq-type nonlinear shallow water long wave equations:

$$
\begin{aligned}
& {\left[1-\left(\mathrm{h}+\frac{\eta}{2}\right)(\nabla \cdot \nabla \eta)-\frac{\mathrm{h}^{3}}{3} \nabla \cdot \nabla(\nabla \cdot \nabla \eta)\right] \frac{\partial \eta}{\partial \mathrm{t}}+} \\
& \nabla \cdot\left[(\mathrm{h}+\eta) \nabla \Phi+\left(\frac{\mathrm{h}^{3}}{3}-\mathrm{h}^{2}-\frac{\mathrm{h}^{3} \eta(\nabla \cdot \nabla \eta)}{3}\right)(\nabla \cdot \nabla \Phi)\right]+ \\
& \left.\frac{J \Phi}{\mathrm{g}}-\left[(\mathrm{h}+\eta) \mathrm{h}+\frac{1}{3} \mathrm{~h}^{3}(2 \mathrm{~h}+\eta) \nabla \cdot \nabla \eta\right)\right] \nabla \cdot \nabla \nabla \cdot \nabla \Phi+ \\
& \nabla(\nabla \cdot \nabla \Phi) \cdot \nabla\left(\frac{\mathrm{h}^{3}}{3}\right)=\mathrm{o}\left(\varepsilon \mu^{4}, \varepsilon^{2} \mu^{2}\right)
\end{aligned}
$$

and

$$
\begin{aligned}
& g \eta+\left(\frac{\partial}{\partial \mathrm{t}}+\mathrm{W}^{*}\right)\left[\Phi-\left(\mathrm{h \eta}+\frac{\eta^{2}}{2}+\frac{\mathrm{h}^{3}}{3} \nabla \cdot \nabla \eta\right)(\nabla \cdot \nabla \Phi)\right]+ \\
& {\left[\frac{1}{2}-\mathrm{h} \nabla \cdot \nabla \eta-\frac{\mathrm{h}^{3}}{3} \nabla \cdot \nabla(\nabla \cdot \nabla \eta)\right](\nabla \Phi \cdot \nabla \Phi)+} \\
& \left(\frac{\mathrm{h}^{2}}{2}+\mathrm{h \eta}\right)(\nabla \cdot \nabla \Phi)^{2}+\left(\frac{\mathrm{h}+\eta}{3}\right) \mathrm{h}^{3} \nabla(\nabla \cdot \nabla \Phi) \cdot \nabla(\nabla \cdot \nabla \Phi) \\
& =\mathrm{o}\left(\varepsilon \mu^{4}, \varepsilon^{2} \mu^{2}\right)
\end{aligned}
$$

\section{Nonlinear long wave equations for very shallow water ( Airy wave )}

when $\mathrm{kh}<<1, \sigma^{2} / g=k^{2} h, c_{g}=c=(g h)^{1 / 2}, k^{2} c c_{g}=g^{2} k h$, the governing equations could be simplified to the Airy wave equations:

$$
\begin{aligned}
& {\left[1+\left(h+\frac{1}{2} \eta\right) k^{2} \eta\right] \frac{D \eta}{D}+\left(\eta+\frac{1}{2} k^{2} m^{2}+\frac{1}{6} k^{2} \eta^{2}\right) \nabla \cdot \stackrel{u}{U}_{c}} \\
& +\nabla \cdot\left[\left(h+\eta+h k^{2} \eta^{2}\right) \nabla \Phi\right]-\left[\frac{J}{g}-k^{4} m(h+\eta)\right] \Phi=0
\end{aligned}
$$

and

$$
\begin{aligned}
& g \eta+\left(\frac{D}{D t}+W\right)\left[\Phi+\left(h+\frac{\eta}{2}\right) \eta k^{2} \Phi\right] \\
& \left.+\left(\frac{1}{2}+h k^{2} \eta\right) \nabla \Phi \cdot \nabla \Phi+\left(\frac{h^{2}}{2}+h \eta\right) k^{4} \Phi^{2}=q t\right)
\end{aligned}
$$

Omitted the high order terms containing $J$ and $k h$ of the above two equations, we can obtain the nonlinear long wave equations for very shallow water(i.e. Airy shallow water long wave equations) as follows: 


$$
\begin{aligned}
& \left(\frac{D}{D}+\nabla \cdot \stackrel{r}{U}_{c}\right) \eta+\nabla \cdot[(h+\eta) \nabla \Phi]=0 \\
& g \eta+\left(\frac{D}{D t}+W^{*}\right) \Phi+\frac{1}{2} \nabla \Phi \cdot \nabla \Phi=C(t)
\end{aligned}
$$

\section{CONCLUSIONS}

1) A fully dispersive fifth order nonlinear wave propagation model for mild current, water level and depth was established theoretically considering energy and topography factors.

2) By omitting the 5th order terms; the lower order model was in consistency with the former 3rd order model.

3)This model could be simplified to mild-slope type equations for deep water, to Boussinesq type equations for shallow water and to Airy wave for very shallow water.

4) The efficiency and accuracy of the mathematical model were yet to be validated.

\section{ACKNOWLEDGEMENT}

The authors wish to acknowledge the support of the national Science Foundation of China (Grant No. Grant No. 51279055; 51579090) and the Fundamental Research Funds for Central Universities (2011B06114).

\section{REFERENCE}

1)Hong G.W., Wu Z.\& Zhang Y. ,2009. Nonlinear mathematical model for Gravity wave propagation on the long wave.[C].The 14th National Symposium on Coastal Engineering,:21 34.

2) Isobe, M., 1994. Time-dependent mild slope equation for random waves. [C]. Japan: Proceeding of the 24th International Conference on Coastal Engineering:285-299.

3) Li B., 2008. Wave equations for regular and irregular water wave propagation. [J]. Journal of waterway, port, coastal and ocean engineering, 134(2):121 142.

4) Nadaoka, K, Beji, S \&Nakagawa, Y. A. , 1994.fully-dispersive nonlinear wave model and its numerical solutions. [C]. Japan: Proceedings of the 24th International Conference on Coastal Engineering:427 441.

5)Zhang Y., 2010a.Fully dispersion nonlinear mathematical wave propagation model and its simulation[D].Nanjing : Hohai University , 2010.

6)Zhang Y., Hong G.W., \& Feng W.B.,2010b.An improved full water nonlinear wave propagation model and its simulation [J]. Journal of Hohai University, 5:592-597. 